\title{
Scoliosis convexity and organ anatomy are related
}

\author{
Tom P. C. Schlösser ${ }^{1} \cdot$ Tom Semple $^{2} \cdot$ Siobhán B. Carr $^{3} \cdot$ Simon Padley $^{2}$ • \\ Michael R. Loebinger ${ }^{4}$ Claire Hogg ${ }^{1,2,3,4} \cdot$ René M. Castelein $^{1}$
}

Received: 24 August 2016/Revised: 1 January 2017/ Accepted: 22 January 2017/Published online: 8 February 2017

(C) The Author(s) 2017. This article is published with open access at Springerlink.com

\begin{abstract}
Purpose Primary ciliary dyskinesia (PCD) is a respiratory syndrome in which 'random' organ orientation can occur; with approximately $46 \%$ of patients developing situs inversus totalis at organogenesis. The aim of this study was to explore the relationship between organ anatomy and curve convexity by studying the prevalence and convexity of idiopathic scoliosis in PCD patients with and without situs inversus.
\end{abstract}

René M. Castelein

r.m.castelein@umcutrecht.nl

Tom P. C. Schlösser

t.p.c.schlosser@umcutrecht.nl

Tom Semple

t.semple@rbht.nhs.uk

Siobhán B. Carr

s.carr@ rbht.nhs.uk

Simon Padley

s.padley@imperial.ac.uk

Michael R. Loebinger

m.loebinger@rbht.nhs.uk

Claire Hogg

c.hogg@rbht.nhs.uk

1 Department of Orthopaedic Surgery, G05.228, University Medical Center Utrecht, P.O. Box 85500, 3508, GA, Utrecht, The Netherlands

2 Department of Radiology, Royal Brompton and Harefield NHS Foundation Trust, London, UK

3 Department of Pediatric Respiratory Medicine, Royal Brompton Hospital, Royal Brompton and Harefield NHS Foundation Trust, London, UK

4 Host Defence Unit, Royal Brompton Hospital, Royal Brompton and Harefield NHS Foundation Trust, London, UK
Methods Chest radiographs of PCD patients were systematically screened for existence of significant lateral spinal deviation using the Cobb angle. Positive values represented right-sided convexity. Curve convexity and Cobb angles were compared between PCD patients with situs inversus and normal anatomy.

Results A total of 198 PCD patients were screened. The prevalence of scoliosis $\left(\mathrm{Cobb}>10^{\circ}\right)$ and significant spinal asymmetry $\left(\mathrm{Cobb} 5-10^{\circ}\right.$ ) was 8 and $23 \%$, respectively. Curve convexity and Cobb angle were significantly different within both groups between situs inversus patients and patients with normal anatomy $(P \leq 0.009)$. Moreover, curve convexity correlated significantly with organ orientation $(P<0.001 ; \phi=0.882)$ : In 16 PCD patients with scoliosis ( 8 situs inversus and 8 normal anatomy), except for one case, matching of curve convexity and orientation of organ anatomy was observed: convexity of the curve was opposite to organ orientation.

Conclusions This study supports our hypothesis on the correlation between organ anatomy and curve convexity in scoliosis: the convexity of the thoracic curve is predominantly to the right in PCD patients that were 'randomized' to normal organ anatomy and to the left in patients with situs inversus totalis.

Keywords Scoliosis - Curve convexity - Situs inversus totalis · Situs solitus · Primary ciliary dyskinesia

\section{Introduction}

Idiopathic scoliosis is a three-dimensional (3-D) deformity of the spine and trunk that primarily affects previously healthy children during the adolescent growth spurt, without any underlying known pathology [1]. A major 
unexplained aspect of idiopathic scoliosis is that the main thoracic spinal curve is most commonly (80-99\%) convex to the right with or without compensatory curves to the left in the high-thoracic and thoraco(lumbar) areas, respectively [2, 3].

A number of factors have been held responsible for the predominance of right-sided thoracic curves in idiopathic scoliosis, in particular asymmetry of the distribution of the thoracic and abdominal organs, brain asymmetry, and the predominance of right handedness in the general population [4].

Incidental reports describe that, in patients with situs inversus totalis or dextrocardia and scoliosis, the curve pattern is the opposite of that found normally: a left convex thoracic curve instead of right convex [5-8]. These reports generate the hypothesis that the convexity of the curve in scoliosis is to the right in patients with normal organ anatomy (situs solitus) and to the left in patients with situs inversus totalis. Information about curve convexity in situs inversus patients could increase our understanding of the factors that are involved in the convexity of idiopathic scoliosis.

The chance of occurrence of idiopathic scoliosis (1:25-50) and situs inversus totalis (1:8000-25,000) in one patient, simultaneously, however, is 1:200,000-1,250,000, making this combination difficult to study in the general population [9-11]. Primary ciliary dyskinesia (PCD) is a disease that is caused by defects in the motile cilia. Since it is associated with bronchiectasis and chronic rhinosinusitis, disease progression is often followed using chest radiography or computed tomography of the thorax. Due to the ciliary defects, during organogenesis, "chance alone will determine whether the viscera will take up the normal or the reversed position" [11]. In contrast to the epidemiology in the normal population, approximately $46 \%$ of PCD patients have situs inversus and the other half is 'randomized' to normal anatomy. In the literature, PCD has not been linked to syndromic scoliosis and the prevalence of idiopathic scoliosis in PCD patients has never been studied.

The aim of this study was (1) to determine the prevalence and convexity of coronal spinal asymmetry and idiopathic scoliosis in PCD patients, and (2) to study the cause-effect relationship between organ anatomy and curve convexity in PCD patients with normal anatomy versus patients with situs inversus totalis or dextrocardia.

\section{Materials and methods}

\section{Study population}

Patients with confirmed diagnosis of PCD in one of four centers of expertise in the United Kingdom between 2013 and 2015 were enrolled. Posterior-anterior chest radiographs taken on clinical grounds or as part of the national annual review program were made according to a standard protocol in the upright position. For all subjects, at least one standard chest radiograph with a laterality ( $\mathrm{L}$ or $\mathrm{R}$ ) marking had to be available. Any available chest CT's were also reviewed. All patients with congenital spinal abnormalities, congenital heart disease, previous (cardio)thoracic surgery in their history, chest radiographs obtained in an external facility, or not obtained in the standing position were excluded.

\section{Scoliosis screening}

The most recent chest radiograph was reviewed by two trained observers in separate settings, blinded to each other's observation. Organ orientation (situs solitus, situs inversus, or dextrocardia) was classified according to the side of the heart and side of the gastric air bubble. For each subject, the observers independently measured the coronal Cobb angles of spinal deviations in the thoracic area using our local PACS system [12]. Mean Cobb angles of the observers were used for further analyses. Positive values corresponded to right-sided curve convexity. A Cobb angle of $0^{\circ}-5^{\circ}$ was defined as no lateral deviation, between $5^{\circ}$ and $10^{\circ}$ as significant lateral deviation and $\geq 10^{\circ}$ as scoliosis (according to the classification of the Scoliosis Research Society) [13].

\section{Statistical analysis}

The statistical analyses were performed using SPSS 22.0 for Windows (SPSS, Inc., Chicago, IL). Descriptive baseline statistics and results were computed for each continuous and ordinal parameter, providing means and standard deviations (SD), and frequencies, respectively. Chi-square test was used to test for statistical differences in curve convexity between subjects with normal anatomy, situs inversus totalis, and dextrocardia. After Levene's test for equality of variances was used, unpaired $t$ tests were used to compare the Cobb angles between the groups. Correlations between curve convexity and organ orientation were analyzed using Phi coefficient. The statistical significance level was set at $<0.05$.

\section{Results}

A total of 256 unique PCD patients were identified. Six were excluded, because no radiographs were available, two because the radiography was obtained in the supine position, and fifty because radiographs had been obtained in external facilities. A total of 198 PCD patients were 
included. Patients were on average $15.3 \pm 12.4$ (median 12.6; range 0-70 years) years old and 109 (55\%) were female. No disagreements on the presence of a coronal curve, organ orientation, or curve convexity existed between the observers. A total of 108 (55\%) subjects had normal organ anatomy (levocardia), 84 (42\%) had situs inversus, and $4(2 \%)$ dextrocardia.

Forty-six (23\%) and sixteen (8\%) subjects had significant lateral deviation of the spine or scoliosis, with a Cobb angle of more than $5^{\circ}$ and $10^{\circ}$, respectively. One patient with situs inversus had undergone scoliosis surgery prior to this study for a left-sided thoracic curve. Scoliosis subjects varied between 2 and 70 years of age. The mean coronal Cobb angle in the lateral deviation group was $6.3^{\circ} \pm 1.7^{\circ}$, and in the scoliosis group, it was $15.6^{\circ} \pm 7.6^{\circ}$. A statistically significant difference in curve convexity was observed between subjects with normal anatomy and situs inversus totalis in both the lateral deviation and the scoliosis group $(P=0.009$ and $P=0.001$, respectively; Tables 1 and 2). There was no scoliosis case in the four dextrocardia patients. In both the lateral deviation and the scoliosis group, the curvature was to the left in the large majority (79-100\%, respectively) of the situs inversus patients and to the right in the normal anatomy group (75-87\%, respectively). In the situs inversus group with scoliosis, Cobb angles varied between $-10^{\circ}$ and $-30^{\circ}$, whereas Cobb angles in the normal anatomy group with scoliosis were between $-10^{\circ}$ and $+16^{\circ}$. Mean Cobb angles were $-19^{\circ} \pm 10^{\circ}$ in subjects with situs inversus with a left convex curve versus $12^{\circ} \pm 2^{\circ}$ in subjects with normal anatomy and a right convex curve $(P<0.001)$. One patient had normal anatomy and a left convex curve of $-10^{\circ}$, and there were no situs inversus patients with a right convex curve. In the lateral deviation group, similar data were found: Situs inversus and left-sided deviation had a mean Cobb angle of $-5^{\circ} \pm 2^{\circ}$ versus situs inversus and right-sided deviation $7^{\circ} \pm 2^{\circ}$ versus normal anatomy and left-sided deviation $-8^{\circ} \pm 1^{\circ}$ versus normal anatomy and right-sided deviation $7 \pm 12$ $(P=0.017)$. Curve convexity correlated significantly with organ orientation in the patients with scoliosis and significant lateral deviation of the spine $(P<0.001 ; \phi$ $=0.882)$.

Table 1 Organ orientation and curve convexity in subjects with significant lateral deviation of the spine (Cobb angle $5^{\circ}-10^{\circ}$ )
Table 2 Organ orientation and curve convexity in subjects with scoliosis (Cobb angle $>10^{\circ}$ )

\begin{tabular}{lll}
\hline & $\begin{array}{l}\text { Left convex } \\
(n=9)\end{array}$ & $\begin{array}{l}\text { Right convex } \\
(n=7)\end{array}$ \\
\hline Normal anatomy $(n=8)$ & $1(13 \%)$ & $7(87 \%)$ \\
Situs inversus $(n=8)$ & $8(100 \%)$ & $0(0 \%)$ \\
\hline
\end{tabular}

Chi-square test, $P=0.001$

\section{Discussion}

Idiopathic scoliosis is a classic and intriguing orthopaedic problem in which the spine collapses into a three-dimensional deformity without a known underlying cause [1]. In its most common form, it is a right convex main thoracic curve with compensatory high-thoracic and (thoraco)lumbar curves to the left $[2,3]$. No good explanation has so far been offered for this typical curve pattern. This study confirms that there is a correlation between organ anatomy and curve convexity in idiopathic scoliosis: In our study population of a mixed group of patients with situs inversus and situs solitus that also have scoliosis, the convexity of the thoracic curve appeared to be predominantly to the right in patients with normal organ anatomy, and to the left in patients with situs inversus (Figs. 1, 2).

To prove causality, it would be better to study a large population with and without situs inversus, without any other pathology, such as PCD. The coincidence, however, of appearance of idiopathic scoliosis (1:25-50) and situs inversus $(1: 8000-25,000)$ in one patient simultaneously is 1:200,000-1,250,000, making such a study almost impossible to carry out [9-11]. We sent an informal written inquiry via the newsletter of the Scoliosis Research Society to a large worldwide group of scoliosis surgeons in 2011 and could not identify any cases with this rare combination.

The clustering of PCD patients in expertise centers and introduction of the annual screening program of pediatric PCD patients in the United Kingdom provided a unique opportunity to perform systematic screening of scoliosis in patients with situs inversus. In the literature, PCD has not been linked to scoliosis and the prevalence of idiopathic scoliosis in PCD patients has never been studied so far. It can be debated, however, whether the increased prevalence

\begin{tabular}{lcc}
\hline & Left convex $(n=15)$ & Right convex $(n=15)$ \\
\hline Normal anatomy $(n=16)$ & $4(25 \%)$ & $12(75 \%)$ \\
Situs inversus $(n=14)$ & $11(79 \%)$ & $3(21 \%)$ \\
\hline
\end{tabular}

Chi-square test, $P=0.009$ 


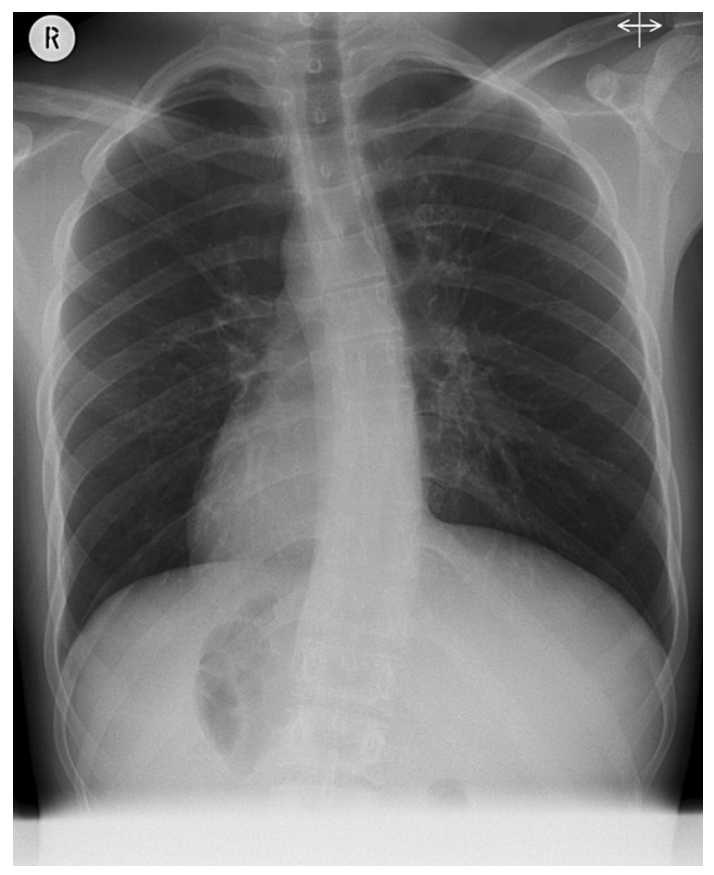

Fig. 1 Example of a primary ciliary dyskinesia patient with situs inversus totalis and a left convex thoracic curve on a chest radiograph

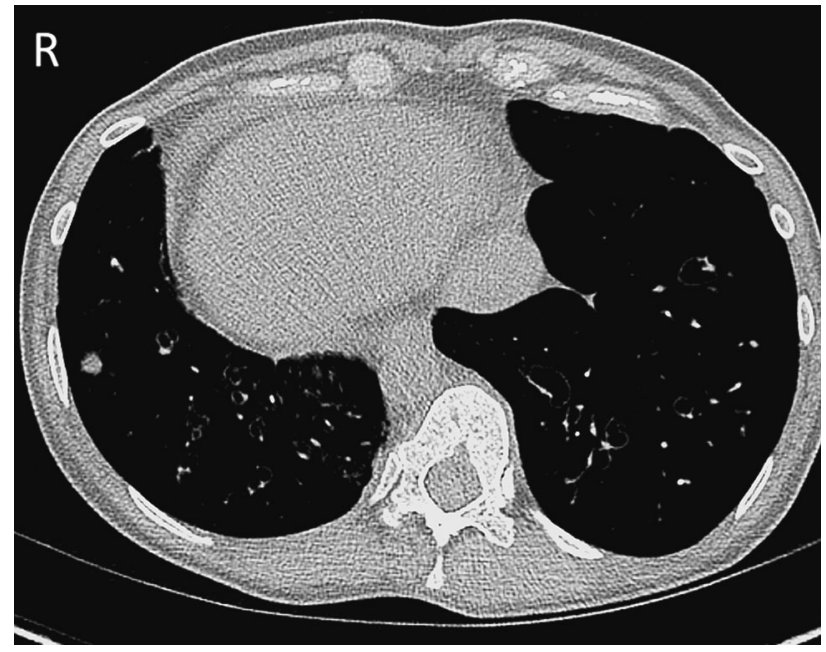

Fig. 2 Transverse image of a computed tomography scan of a 25-year-old patient affected by both primary ciliary dyskinesia patient and scoliosis, demonstrating the internal relation between positioning of the heart and aorta and spinal rotation. Even on soft tissue window settings, at the right lung base, results of their PCD are visible, e.g., bronchiectasis and mucus plugging

of scoliosis, (8\%) in this PCD population versus $1.5-3 \%$ in the normal population, suggests predisposition to the development of a spinal deformity in PCD [14]. However, in other epidemiological studies, the prevalence of thoracic scoliosis on chest radiographs of asymptomatic adults is $8-24 \%$ [15-17]. On the one hand, the prevalence provided by our study may underestimate the prevalence of scoliosis in PCD, because a minority of our population was still prepubertal, $31 \%$ of our population was under the age of ten. Recently, Oliazadeh et al. found abnormalities in osteoblast primary cilia in idiopathic scoliosis patients and Grimes et al. have demonstrated a developmental zebra-fish model that suggests a link between ependymal cell cilia development and the onset of scoliosis [18, 19]. On the other hand, a pilot study on 48 PCD patients in The Netherlands did not result in retrieval of scoliosis cases, and in other pediatric pulmonary diseases, such as cystic fibrosis, a scoliosis prevalence of $2-11 \%$ has been described [20, 21]. Based on the curve pattern and clinical presentation in our study of PCD patients, all curves were considered to be of the idiopathic-type, but selection bias cannot be excluded.

As has been appreciated for a long time, the normal spine is not a symmetrical structure. Tallroth et al. demonstrated the relation between coronal spinal alignment and dextrocardia in a population without scoliosis [8]. Moreover, in the previous studies by Janssen et al. and Kouwenhoven et al., it was observed that the normal, nonscoliotic thoracic spine of adolescents and adults is slightly rotated to the right pre-existently and that this corresponds to asymmetrical closure of the neurocentral junctions [22, 23]. In contrast, on MRI scans in a group of nonscoliotic subjects with PCD and situs inversus, a rotational pattern exactly opposite to what was found in the normal adult population was demonstrated [24]. Despite the mirrored orientation of the internal organs, handedness was distributed as in the general populationthe vast majority was right-handed. Therefore, it was concluded that handedness does not influence the preexistent rotational patterns of the normal spine, but organ anatomy may.

Interestingly, Janssen et al. also found that the direction of this pre-existent rotation of the spine changes direction between the infantile and adolescent age. No explanation was offered, but this suggests a change in the distribution of internal mass during development [22, 23]. The relation between handedness and organ orientation in PCD patients has also previously been investigated by McManus et al. They observed no significant differences in handedness for writing between situs inversus and situs solitus patients [25]. In accordance with the previous observation, our present study suggests that organ orientation in the body plays an important role in determining the direction of spinal curvature. Once spinal decompensation occurs, its direction is determined by the distribution of internal organ anatomy. Because of the crosssectional design, with a pseudo-randomized, and due to the rarity of disease a limited sample size, our study may not provide definite information about cause-and-effect, but only can demonstrate a certain relation between the two parameters. 


\section{Conclusions}

This study confirms that the convexity of the curve in idiopathic scoliosis is predominantly to the right in patients with normal organ anatomy (situs solitus), and to the left in patients with situs inversus totalis. This matches with the previous observation that pre-existent rotation of the spine correlates with organ anatomy and with the hypothesis that once the spine decompensates into an idiopathic scoliosis, it will follow its pre-existent rotational pattern.

Acknowledgements Funding was provided by K2M unrestricted research grant and AOSpine (Young Researcher Award 2015).

\section{Compliance with ethical standards}

Conflict of interest Tom Schlösser received a personal research grant by AOSpine. Michael Loebinger is a paid advisory board member of Insmed, Bayer, Pumocide, Raptor and Chiesi and a consultant of Insmed. René Castelein received a research grant by and is a paid consultant for K2M. Tom Semple, Siobhan Carr and Claire Hogg have no potential conflict of interest.

Ethical approval This study was approved by the local medical ethical review boards.

Open Access This article is distributed under the terms of the Creative Commons Attribution 4.0 International License (http://crea tivecommons.org/licenses/by/4.0/), which permits unrestricted use, distribution, and reproduction in any medium, provided you give appropriate credit to the original author(s) and the source, provide a link to the Creative Commons license, and indicate if changes were made.

\section{References}

1. Cheng JC, Castelein RM, Chu WC, Danielsson AJ, Dobbs MB, Grivas TB, Gurnett CA, Luk KD, Moreau A, Newton PO, Stokes IA, Weinstein SL, Burwell RG (2015) Adolescent idiopathic scoliosis. Nat Rev Dis Primers 1:15030. doi:10.1038/nrdp.2015. 30

2. Lenke LG, Betz RR, Harms J, Bridwell KH, Clements DH, Lowe TG, Blanke K (2001) Adolescent idiopathic scoliosis: a new classification to determine extent of spinal arthrodesis. J Bone Jt Surg Am 83-A:1169-1181

3. Parent S, Newton PO, Wenger DR (2005) Adolescent idiopathic scoliosis: etiology, anatomy, natural history, and bracing. Instr Course Lect 54:529-536

4. Kouwenhoven JW, Castelein RM (2008) The pathogenesis of adolescent idiopathic scoliosis: review of the literature. Spine 33:2898-2908

5. Tanaka K, Sutani A, Uchida Y, Shimizu Y, Shimizu M, Akita M (2007) Ciliary ultrastructure in two sisters with Kartagener's syndrome. Med Mol Morphol 40:34-39. doi:10.1007/s00795007-0354-y

6. Engesaeth VG, Warner JO, Bush A (1993) New associations of primary ciliary dyskinesia syndrome. Pediatr Pulmonol 16:9-12

7. Burwell RG, Dangerfield PH, Freeman BJ, Aujla RK, Cole AA, Kirby AS, Pratt RK, Webb JK, Moulton A (2006) Etiologic theories of idiopathic scoliosis: the breaking of bilateral symmetry in relation to left-right asymmetry of internal organs, right thoracic adolescent idiopathic scoliosis (AIS) and vertebrate evolution. Stud Health Technol Inform 123:385-390

8. Tallroth K, Lohman M, Heliovaara M, Aromaa A, Knekt P, Standertskjold-Nordenstam CG (2009) Dextrocardia and coronal alignment of thoracic curve: a population study. Eur Spine J 18:1941-1945. doi:10.1007/s00586-009-1049-y

9. Altaf F, Gibson A, Dannawi Z, Noordeen H (2013) Adolescent idiopathic scoliosis. BMJ 346:f2508. doi:10.1136/bmj.f2508

10. Afzelius BA (1995) Situs inversus and ciliary abnormalities. What is the connection? Int J Dev Biol 39:839-844

11. Pennekamp P, Menchen T, Dworniczak B, Hamada H (2015) Situs inversus and ciliary abnormalities: 20 years later, what is the connection? Cilia 4:1. doi:10.1186/s13630-014-0010-9

12. Cobb JR (1948) Outline for the study of scoliosis. Instr Course Lect Am Acad Orthop Surg 5:261-275

13. Working Group on 3-D Classification (Chair Larry Lenke, MD), and the Terminology Committee (2000) SRS Terminology Committee and Working Group on Spinal Classification Revised Glossary of Terms. http://www.srs.org/professionals/online-edu cation-and-resources/glossary/revised-glossary-of-terms. Accessed 4 Nov 2016

14. Lonstein JE (1994) Adolescent idiopathic scoliosis. Lancet 344:1407-1412

15. Carter OD, Haynes SG (1987) Prevalence rates for scoliosis in US adults: results from the first National Health and Nutrition Examination Survey. Int J Epidemiol 16:537-544

16. Urrutia J, Zamora T, Klaber I (2014) Thoracic scoliosis prevalence in patients 50 years or older and its relationship with age, sex, and thoracic kyphosis. Spine 39:149-152. doi:10.1097/BRS. 0000000000000095

17. Chen JB, Kim AD, Allan-Blitz L, Shamie AN (2016) Prevalence of thoracic scoliosis in adults 25 to 64 years of age detected during routine chest radiographs. Eur Spine J 25:3082-3087. doi:10.1007/s00586-015-4215-4

18. Oliazadeh N, Franco A, Wang D, Moreau A (2015) Abnormalities in primary cilium of osteoblasts of adolescent idiopathic scoliosis patients. Cilia 4(Suppl 1):6

19. Grimes DT, Boswell CW, Morante NF, Henkelman RM, Burdine RD, Ciruna B (2016) Zebrafish models of idiopathic scoliosis link cerebrospinal fluid flow defects to spine curvature. Science 352:1341-1344. doi:10.1126/science.aaf6419

20. Fainardi V, Koo SD, Padley SP, Lam SH, Bush A (2013) Prevalence of scoliosis in cystic fibrosis. Pediatr Pulmonol 48:553-555. doi:10.1002/ppul.22624

21. Hathorn C, Fall A, McGurk S, Tsirikos AI, Urquhart DS (2014) Acquisition bias may have led to acceptance of the false null hypothesis that prevalence of scoliosis is the same in cystic fibrosis as the general population. Pediatr Pulmonol 49:201. doi:10.1002/ppul.22947

22. Janssen MM, Kouwenhoven JW, Schlosser TP, Viergever MA, Bartels LW, Castelein RM, Vincken KL (2011) Analysis of preexistent vertebral rotation in the normal infantile, juvenile, and adolescent spine. Spine 36:E486-E491. doi:10.1097/BRS. 0b013e3181f468cc

23. Kouwenhoven JW, Vincken KL, Bartels LW, Castelein RM (2006) Analysis of preexistent vertebral rotation in the normal spine. Spine 31:1467-1472. doi:10.1097/01.brs.0000219938. 14686.b3

24. Kouwenhoven JW, Bartels LW, Vincken KL, Viergever MA, Verbout AJ, Delhaas T, Castelein RM (2007) The relation between organ anatomy and pre-existent vertebral rotation in the normal spine: magnetic resonance imaging study in humans with situs inversus totalis. Spine 32:1123-1128

25. McManus IC, Martin N, Stubbings GF, Chung EM, Mitchison HM (2004) Handedness and situs inversus in primary ciliary dyskinesia. Proc Biol Sci 271:2579-2582 